\title{
Appreciation to reviewers
}

Published online: 10 February 2022

(C) The Author(s), under exclusive licence to Senckenberg Gesellschaft für Naturforschung 2022

Marine Biodiversity has concluded its $51^{\text {st }}$ volume of publication. I am very grateful to all authors, reviewers, and Editorial Board members for their exceptional contributions to this peer-reviewed publication, particularly during this COVID19 Global Pandemic. They all provide an immeasurable wealth of expertise and willingness to volunteer their time and vivacity on behalf of Marine Biodiversity.
I would like to express my deepest gratitude to all members of the Editorial Board and to the manuscript authors.

I would like to especially thank and express my deepest appreciation to the individuals listed below, all of whom kindly reviewed manuscripts submitted to Marine Biodiversity during 2021.

Pedro Martínez Arbizu

Editor-in-Chief

Marine Biodiversity

Pere Abelló

Lutfi Afiq-Rosli

Rosalía Aguilar Medrano

K.V. Akhilesh

Edgar Alcántara-Razo

Felipe Amezcua

Frank E. Anderson

Jonathan W. Armbruster

Maickel Armenteros

Werner Armonies

Akira Asakura

Elisabeth C. Ashton

Gail Ashton

Massimo Avian

Bonnie A. Bain

Carlos A.M. Barboza

Levent Bat

Sarah Beauvais

Giambattista Bello

Hans Bertsch

Rebecca J. Best

James A. Blake

María del Pilar Blanco-Parra

Ekaterina V. Bogomolova

Arthur R. Bos

Christopher B. Boyko

Simone Nunes Brandão
Georg Brenneis

Bastian Brenzinger

Christian Briseño-Avena

Ciemon Caballes

Andrea A. Cabrera

Stephen D. Cairns

Pierluigi Carbonara

Alvar Carranza

Joan Enric Cartes

Tin-Yam Chan

Wei-Jen Chen

Qiqun Cheng

Anne Chenuil

Yves Cherel

Andrew T.M. Chin

Cecilia G. Conaco

Brandon J. Conroy

Paulo Henrique Costa Corgosinho

Jorge Cortés

Jenna M. Crowe-Riddel

Victor Hugo Cruz-Escalona

Tauana Junqueira Cunha

Daphne Cuvelier

Padmini Dalpadado

Giulia Dapueto

José De La Cruz-Agüero

Thiago Silva de Paula
Renate Degen

Andrea Desiderato

Flávio Dias Passos

Lars Christian Dietz

Cláudia Câmara do Vale

Martin Dohrmann

Marie Drabkova

Jennifer M. Durden

Douglas Eernisse

Joshua P. Egan

Marc Pierre Eléaume

Jim R. Ellis

Ahmed El-Shafei

Hiromitso Endo

Deniz Ergüden

Hamid Reza Esmaeili

Gal Eyal

Doug Fenner

Fernando Fernández-Álvarez

Sergio Flores-Ramírez

Charles H.J.M. Fransen

Giulia Furfaro

John Galbraith

Jaaziel Emmanuel Garcia-Hernandez

Manuel Roberto Garcia-Huidobro

Kai Horst George

Klaas Gerdes 
Damian Gaspar Gil

Chris Glasby

Serge Gofas

Alexey V. Golikov

Jaime Alberto Jardim Gomes

Paula Braga Gomes

Saúl González-Murcia

Dennis Preston Gordon

Terrence Gosliner

Tricia C. Goulding

Charles Llewellyn Griffiths

Dean Grubbs

Ángel Guerra

James Guest

Lars Gutow

Takuma Haga

Jakob Hallermann

Roger Hanlon

Muge Aliye Hekimoglu

Alicia Hermosillo

Junya Hirai

Shimpei Hiruta

Bert W. Hoeksema

Leon Hoffman

Ivona Horká

Aino Hosia

Shannon Hsieh

Danwei Huang

Wen-Chien Huang

Kevin David Hyde

Tohru Iseto

Hajime Ishihara

Rima W. Jabado

Ashok Kumar Jaiswar

Ronald Janssen

Anna Maria Jażdżewska

Erika Johnston

Elaina Jorgensen

Heather Judkins

Carly Kenkel

Shashank Keshavmurthy

Sahar Khodami

Matthew Kimball

Seishi Kimura

Hiroki Kise

Marcelo Visentini Kitahara

Toru Kobari

Gregory A. Kolbasov

Ingrid Kröncke

Svjetlana Krstulović Šifner

Patrick Krug

Tsunemi Kubodera

Piotr Kuklinski
Yuka Kushida

Peter M. Kyne

Raul Laiz-Carrión

Jannes Landschoff

Tom Langbehn

Vladimir Laptikhovsky

Vincent Laudet

Sébastien Lavoué

Jonathan W. Lawley

John M. Lawrence

Eric Lazo-Wasem

Xinzheng Li

Laura Elizabeth Lilly

Dhugal Lindsay

Marina Loeb

Caterina Longo

Cataixa López

Pablo López González

Lara Macheriotou

Enrique Macpherson

Davide Maggioni

Alex Maile

Antônio João Malafaia Peixoto

Emanuele Mancini

Ivan Marin

J. Fernando Márquez-Farías

Luis Martell

Raul Martínez-Rincón

Rafaqat Masroor

Luz Elena Mateo-Cid

Jennifer Mather

Francesc Maynou

Ana Carolina Mazzuco

Linda McCann

Catherine McFadden

Karin Meißner

Nuría Méndez

Manuel Mendoza-Carranza

Angela Mendoza-González

Alex E. Mercado-Molina

Charles G. Messing

Miguel Mies

Hiroshi Miyake

Anil Mohapatra

Alec Moore

Uzi Motro

Marta Moyano

Paul Retson Muir

Jaruwat Nabhitabhata

Kenta Nakamoto

Yohei Nakamura

Rie Nakano

Tohru Naruse
Gavin J.P. Naylor

Mei Lin Neo

Birger Neuhaus

Jessika M. M. Neves

Claus Nielsen

Sofía Ortega García

Masayuki Osawa

Lenin Enrique Oviedo Correa

Paulo Cesar Paiva

Ferran Palero

Per J. Pallsboll

Yannis Papastamatiou

Annam Pavan-Kumar

Rachael A. Peart

Carlos Daniel Perez

Arturo Acero Pizarro

Angelo Poliseno

Marianne Porter

Morgan Pratchett

Günter Purschke

Declan Quigley

Vincent Raoult

Dina Rasquinha

Amanda Reid

James Davis Reimer

José Amorim Reis-Filho

Ana Riesgo

Mohammed Rizman-Idid

Rosana Rocha

Axayacatl Rocha-Olivares

Chiara Romano

Marta Ronowicz

Héloïse Rouzé

Marta Rufino

Gorgonio Ruiz-Campos

Jodie Rummer

Romain Sabroux

Chester Sands

Omar Santana-Morales

Nadiezhda Santodomingo

Cinthya Santos

Maria Eduarda Alves Santos

Laura Schejter

Nikolaos V. Schizas

Anja Schulze

Enrico Schwabe

Tifeng Shan

Elizabeth K. Shea

Craig Sherman

Guelson Batista Silva

Luiz Ricardo Simone

$\mathrm{Na}$ Song

Michael Spector 
Nir Stern

Nicolas Straube

Stephen Summers

Zhong-Min Sun

Kazutaka Takahashi

Tsuyoshi Takano

Agnieszka Tatarek

Paul Taylor

Manuel Tenorio

Delphine Thibault

Patricia Thibodeau

Ekin Tilic

Francesco Tiralongo

Anna Törnroos

Paul Valentich-Scott

Fausto Valenzuela-Quiñonez

Luis Artur Valões Bezerra

Rebecca Varney
Michael Vecchione

Virag Venekey

Carlos Renato Rezende Ventura

Alessandra Viana

Saverio Vicario

Benjamin Victor

Héctor Villalobos

Samuel Vohsen

Janet Voight

Oliver Voigt

Khor Waiho

Lexie Margaret Walker

Anders Warén

Andreas Waser

Mathias Wegner

Ornella Weideli

Simon Weigmann

Sofia Wikström
Gary C. Williams

Jason D. Williams

Inger Eleanor Winkelmann

Judith Winston

Nur Leena Wong

Yuichiro Yamada

Atsushi Yamaguchi

Telat Yanik

Nicholas Yap

Ryuta Yoshida

Mikołaj Zapalski

Salvador Zarco-Perello

Argyro Zenetos

Dongsheng Zhang

Shuqian Zhang

Guoping Zhu

Adriana Zingone

Publisher's note Springer Nature remains neutral with regard to jurisdictional claims in published maps and institutional affiliations. 